\title{
An Ocimum basilicum Extract Containing Rosmarinic Acid Restores the Disruption of Collagen Fibers Caused by Repetitive UVA Irradiation of Dermal Fibroblasts
}

\author{
Madoka Yoshikawa, Yuri Okano, and Hitoshi Masaki* \\ Tokyo University of Technology, 1404-1 Katakura-machi, Hachioji-shi, 192-0982 Tokyo, JAPAN
}

\begin{abstract}
Photoaged skin is characterized by the appearance of pigmented spots such as solar lentigos, deep wrinkles and sags, and progresses due to chronic sun exposure. Among the wavelengths of sunlight, UVA is responsible for the appearance of wrinkles and sags that originate from structural alterations in the dermis of photoaged skin such as the depletion of collagen fibers. Thus, improving and restoring collagen fibers is an effective approach to reduce skin photoaging and maintain a youthful appearance. This study was conducted to evaluate the potential of an extract of Ocimum basilicum (OC), which contains rosmarinic acid (RA), as an anti-photoaging material focusing on the capacity to restore collagen fibers that are disrupted due to intracellular oxidative stress. In spite of their relatively low capacities for chemical scavenging of reactive oxygen species (ROS), both OC and RA showed efficient removal of biological oxidative stress by reducing levels of intracellular ROS and carbonylated proteins (CPs) in fibroblasts following exposure to single or repetitive UVA irradiations. Fibroblasts irradiated with repetitive UVA as a model for chronic sunexposed cells showed significant increases in matrix metalloproteinase-1 and decreases in type I collagen synthesis and formed reduced numbers of collagen fibers. Since both $O C$ and RA restored the adverse phenomena caused by repetitive UVA irradiation, we conclude that OC containing RA is an effective antiphotoaging material.
\end{abstract}

Key words: UVA, collagen fiber, MMP-1, fibroblast

\section{Introduction}

It is well known that excessive UV exposure injures the skin as acute reactions such as causing inflammation and subsequent increases in wrinkles and sags. Although damage caused by UVB is limited to specific regions of the epidermis due to its limited penetration depth into the skin, UVA affects the homeostasis of the dermal matrix, including the cellular functions of fibroblasts, due to its deeper penetration into the dermis ${ }^{1)}$. Thus, chronic UVA exposure of the skin is responsible for causing wrinkles and sags, which are typical symptoms of skin aging termed photoaging. The structural alterations of collagen fibers and elastic fibers that are caused by chronic UVA exposure cause fragility in the dermal matrix and result in photoaging of the $\operatorname{skin}^{2,3)}$. In photoaged skin, collagen fibers, which are major fibers in the dermis, are depleted in the papillary dermis due to an imbalance of their synthesis and degrada- tion $^{4,5)}$. Matrix metalloproteinases (MMPs) are responsible for the degradation of collagen molecules. Among MMPs, MMP-1, which decomposes fibrous collagen initially, is an important enzyme of collagen degradation, and is present at high levels in UV exposed skin ${ }^{6}$. These facts indicate the importance of maintaining collagen fiber structure and eliminating the harmful actions of chronic UVA to prevent wrinkling and sagging of the skin.

The level of MMP-1 in sun-exposed skin has been well demonstrated to be regulated by reactive oxygen species $(\mathrm{ROS})^{7)}$. In general, it is also well recognized that UVA is a potent intracellular generator of ROS. UVA generates singlet oxygen $\left({ }^{1} \mathrm{O}_{2}\right)$ through a photosensitizing reaction ${ }^{8)}$ and also superoxide anion radicals $\left(\cdot \mathrm{O}_{2}{ }^{-}\right)$through the activation of NADPH oxidase ${ }^{9)}$. Meanwhile, carbonylated proteins (CPs) are present at a higher frequency in the upper dermis of the sun-exposed skin of elderly subjects.

\footnotetext{
*Correspondence to: Hitoshi Masaki, Tokyo University of Technology, 1404-1 Katakura-machi, Hachioji-shi, 192-0982 Tokyo, JAPAN E-mail: masaki@stf.teu.ac.jp Accepted August 18, 2020 (received for review May 14, 2020) Journal of Oleo Science ISSN 1345-8957 print / ISSN 1347-3352 online http://www.jstage.jst.go.jp/browse/jos/ http://mc.manusriptcentral.com/jjocs
} 
CPs are synthesized by reactions between amino groups in proteins and aldehyde compounds yielded by lipid peroxidation initiated by ROS. Thus, CPs are regarded as a footprint of higher oxidation levels. Furthermore, our recent study reported that CPs enhance the biosynthesis of MMP-1 through the elevation of intracellular $\mathrm{ROS}^{10)}$. These facts indicate the importance of reducing levels of ROS in order to ameliorate the deleterious changes of collagen mediated by UVA. Furthermore, it is also important to evaluate the efficacy of materials using fibroblasts exposed to repetitive UVA as a model for fibroblasts in photoaged skin, because photoaged skin has changes in appearance caused by chronic UV exposure.

Ocimum basilicum (OC), which contains rosmarinic acid (RA) as a major component, is generally known as sweet basil. OC has been utilized as a traditional medicine, and recent studies have reported its efficacy as an antioxidant $^{11)}$. Furthermore, RA suppresses levels of ROS in HaCaT keratinocytes that are elevated by UVB through the activation of Nrf2 signals $^{12)}$. These facts suggest that OC containing RA would have a potent capability to improve or prevent skin photoaging by restoring the cellular functions of fibroblasts altered by chronic UVA exposure.

Thus, the aim of this study was to clarify the potential of $\mathrm{OC}$ and RA as anti-photoaging materials focusing on the restoration of the formation of collagen fibers affected by repetitive UVA irradiation vis the reduction of intracellular ROS levels.

\section{Materials and Methods \\ 2.1 Materials}

The Ocimum basilicum extract (OC) which was extracted with $50 \%$ ethanol aqueous solution, was a gift from ABResearch srl Via dell'Impresa (Brendola(VI) Italy). Tris (hydroxymethyl) aminomethane, hydrochloric acid, $\mathrm{H}_{2} \mathrm{O}_{2}$, $L$-ascorbic acid, DL- $\alpha$ tocopherol, paraformaldehyde, phosphate buffer solution (PBS), sodium azide $\left(\mathrm{NaN}_{3}\right)$ and xanthine oxidase (XOD) were purchased from Nacalai tesque (Kyoto, Japan). RA, iron (II) perchlorate $\left(\mathrm{Fe}\left(\mathrm{ClO}_{4}\right)_{2}\right)$, 2,2'azino-bis (3-ethylbenzothiazoline-6-sulfonic acid) diammonium salt (ABTS) and hypoxanthine (HPX) were obtained from FUJIFILM Wako Pure Chemical Corp. (Osaka, Japan). 5,5-Dimethyl-1-pyrroline N-oxide (DMPO), tert-butyl hydroperoxide $(t-\mathrm{BuOOH})$, diethylenetriamine-N,N,N',N",N"pentaacetic acid (DTPA) and 2,2,6,6-tetramethyl-4-piperidone hydrochloride (TMPD) were purchased from Tokyo Chemical Industry (Tokyo, Japan). 2-Morpholinoethanesulfonic acid, monohydrate (MES) was obtained from Dojindo (Kumamoto, Japan). Hematoporphyrin (HP) was obtained from MP Biomedicals (CA, USA). Peroxidase and ReverTra Ace qPCR Master Mix were obtained from TOYOBO (Osaka, Japan). Triton X-100 reduced and bovine serum albumin
(BSA) were obtained from Sigma-Aldrich (MO, USA). $\mathrm{H}_{2}$ DCFDA was obtained from Merck (Darmstadt, Germany). The BCA protein assay kit was obtained from Thermo Fisher Scientific (MA, USA). Fluorescein-5-thiosemicarbazine (FTSC) and Hoechst 33342 were obtained from Invitrogen Corp. (CA, USA). Anti-MMP-1 antibody (goat polyclonal)was obtained from Gene Tex (CA, USA). Anticollagen type I antibody conjugated to biotin (rabbit polyclonal)was obtained from Rockland Scientific (B.C., Canada). Anti-goat IgG antibody conjugated to HRP and streptavidin conjugated to HRP were obtained from R\&D Systems (MN, USA). Ez WestBlue was obtained from ATTO (Tokyo, Japan). Anti-rabbit $\operatorname{Ig} G(\mathrm{H}+\mathrm{L}), \mathrm{F}\left(\mathrm{ab}{ }^{\prime}\right)_{2}$ fragment (Alexa Fluor 594 conjugate) was obtained from Cell Signaling (MA, USA).

\subsection{Chemical scavenging capability of $O C$ and RA against superoxide anion radicals $\left(\cdot \mathrm{O}_{2}^{-}\right)$, hydroxyl radicals $(\cdot \mathrm{OH})$, lipid peroxyl radicals $(\mathrm{LOO} \cdot)$ and sin- glet oxygen $\left({ }^{1} \mathrm{O}_{2}\right)$}

Scavenging against ROS was measured by the ESR spintrapping method using a RFR-30 spectrometer (Radical Research, Tokyo, Japan).

Scavenging against $\cdot \mathrm{O}_{2}{ }^{-}$was carried out using a mixture of $\mathrm{XOD}(0.19 \mathrm{U} / \mathrm{mL})$ and $\mathrm{HPX}(0.07 \mathrm{mM})$ as a source of $\cdot \mathrm{O}_{2}{ }^{-}$, and DMPO as a spin trapping agent. At 1 min after mixing $0.1 \mathrm{M}$ phosphate buffer ( $\mathrm{pH} 7.4$ ) containing $70 \mu \mathrm{M}$ HPX, 7 $\mu \mathrm{M}$ DTPA and $90 \mathrm{mM}$ DMPO with $0.19 \mathrm{U} / \mathrm{mL}$ XOD, monitoring of the ESR spectrum was started using the ESR conditions detailed below.

Scavenging against $\cdot \mathrm{OH}$ was carried out using $\mathrm{H}_{2} \mathrm{O}_{2}$ and $\mathrm{Fe}\left(\mathrm{ClO}_{4}\right)_{2}$ as a source of $\cdot \mathrm{OH}$ and $\mathrm{DMPO}$ as a spin trapping agent. At $1 \mathrm{~min}$ after mixing deionized $\mathrm{H}_{2} \mathrm{O}$ containing 40 $\mu \mathrm{M} \mathrm{Fe}\left(\mathrm{ClO}_{4}\right)_{2}, 1.18 \mathrm{mM}$ DTPA, $90 \mathrm{mM}$ DMPO and $160 \mu \mathrm{M}$ $\mathrm{H}_{2} \mathrm{O}_{2}$, monitoring of the ESR spectrum was started using the ESR conditions detailed below.

Scavenging against $\mathrm{LOO} \cdot$ was carried out using $t$ - $\mathrm{BuOOH}$ and $\mathrm{Fe}\left(\mathrm{ClO}_{4}\right)_{2}$ as a source of $\mathrm{LOO} \cdot$ and DMPO as a spin trapping agent. At 1 min after mixing deionized $\mathrm{H}_{2} \mathrm{O}$ containing $480 \mu \mathrm{M} \mathrm{Fe}\left(\mathrm{ClO}_{4}\right)_{2}, 1.18 \mathrm{mM}$ DTPA, $90 \mathrm{mM}$ DMPO and $1.9 \mathrm{mM} t$-BuOOH, monitoring of the ESR spectrum was started using the ESR conditions detailed below.

Scavenging against ${ }^{1} \mathrm{O}_{2}$ was measured using TMPD as a spin trapping reagent and $\mathrm{HP}$ as a photosensitizer to generate ${ }^{1} \mathrm{O}_{2}$ under UVA irradiation. After mixing $25 \mathrm{mM}$ TMPD, $0.1 \mathrm{mM} \mathrm{HP}$ and sample in $0.1 \mathrm{M}$ Tris/HCl $(\mathrm{pH} \mathrm{8.5)}$, the mixture was transferred into a glass capillary set in a cavity of ESR. After UVA irradiation for $45 \mathrm{~s}\left(3.5 \mathrm{~J} / \mathrm{cm}^{2}\right)$, monitoring of the ESR spectrum was started using the following ESR conditions.

ESR spectra were measured with the following conditions; output power: $4.00 \mathrm{~mW}$, scanning field: $336.5 \mathrm{mT} \pm$ $5.0 \mathrm{mT}$, modulation frequency: $0.20 \mathrm{mT}$, response time: 0.03 s. In all measurements, the intensity of Mn(II) doped in 
MnO was monitored as an external standard. Each ROS intensity was expressed as a value in which the signal intensity originating from each ROS was normalized with the signal intensity of $\mathrm{Mn}^{2+}$. The scavenging capability against each type of ROS is expressed as an $\mathrm{IC}_{50}$, which is the $50 \%$ scavenging concentration. $\mathrm{IC}_{50}$ 's of $\mathrm{NaN}_{3}\left(\right.$ for $\left.{ }^{1} \mathrm{O}_{2}\right), L$-ascorbic acid (for $\cdot \mathrm{O}_{2}{ }^{-}$and $\cdot \mathrm{OH}$ ) and DL- $\alpha$-tocopherol (for $\mathrm{LOO} \cdot$ ) were measured as a positive control for each type of ROS.

\subsection{Cell culture}

Normal human dermal fibroblasts (NHDFs) (Kurabo, Osaka, Japan) were cultured in Dulbecco's modified Eagle medium (DMEM) (Nissui, Tokyo, Japan), supplemented with $5 \%$ fetal bovine serum (FBS) (Biowest, Nuaillé, France) and incubated in a humidified atmosphere of $95 \%$ air and $5 \% \mathrm{CO}_{2}$ at $37^{\circ} \mathrm{C}$.

\subsection{UVA irradiation}

UVA irradiation was carried out using a FL20/BLB fluorescent lamp (Toshiba, Tokyo, Japan), which has a radiation peak at $352 \mathrm{~nm}$, as the UVA light source. The irradiation energy was measured with an UVP UVX Radiometer: UVX-25 Sensor (Fisher Scientific, CA, USA). Cells were irradiated with UVA in Hanks' buffered salt solution containing $\mathrm{Mg}^{2+}$ and $\mathrm{Ca}^{2+}$ (HBSS).

\subsection{Biological anti-oxidative effects of $O C$ and RA in NHDFs irradiated by UVA}

NHDFs were seeded at a density of $2 \times 10^{4}$ cells/well in 96 well plates and then were loaded with $20 \mu \mathrm{M} \mathrm{H}_{2}$ DCFDA for $30 \mathrm{~min}$. Cells irradiated with UVA at a dose of $10 \mathrm{~J} / \mathrm{cm}^{2}$ were incubated in HBSS in the absence or presence of OC or RA for $6 \mathrm{~h}$. After washing with HBSS, the fluorescence intensity (F.I.) (Ex: 485 nm, Em: 530 nm) of cells was measured in order to determine intracellular ROS levels. Protein contents were quantified with the BCA protein assay kit after lysing with PBS (phosphate-buffered saline without $\mathrm{Ca}^{2+}$ and $\mathrm{Mg}^{2+}$ ) containing $0.5 \%$ Triton X-100. Intracellular ROS levels were calculated as F.I./ $\mu$ g protein and are expressed as a fold change against the intracellular ROS level of untreated sham-UVA irradiated NHDFs. Levels of CPs in cells were examined as follows: NHDFs were exposed to UVA at a dose of $10 \mathrm{~J} / \mathrm{cm}^{2}$ once a day for 1 $\mathrm{d}$ or for 3 successive days. NHDFs were cultured in DMEM containing $5 \%$ FBS in the presence or absence of OC or RA for $24 \mathrm{~h}$ after each UVA irradiation. At $24 \mathrm{~h}$ after the last UVA irradiation, intracellular CPs were fluorescencelabeled with $20 \mu \mathrm{M}$ FTSC in 0.1 M MES Na buffer (pH 5.5) for $1 \mathrm{~h}$ in the dark. Nuclei of cells were stained with $2 \mu \mathrm{M}$ Hoechst 33342. The F.I. originating from CPs (Ex: $465 \mathrm{~nm}$ Em: $535 \mathrm{~nm}$ ) and from nuclei (Ex: $350 \mathrm{~nm}$; Em: $461 \mathrm{~nm}$ ) was measured with a SpectraMax Gemini EM Microplate Reader (Molecular Devices, CA, USA). Intracellular CPs levels were calculated as F.I. derived from FTSC labeling/ F.I. derived from Hoechst 33342, and are expressed as fold change normalized with intracellular CPs levels of untreated sham-UVA irradiated NHDFs. In addition, fluorescence images were obtained using a Floid Cell Imaging Station (Life Technology, CA, USA).

\subsection{Real-time RT PCR analysis}

NHDFs were irradiated with UVA at a dose of $10 \mathrm{~J} / \mathrm{cm}^{2}$ once a day for 3 successive days. NHDFs were cultured in DMEM containing 5\% FBS in the presence or absence of OC or RA for $24 \mathrm{~h}$ after each UVA irradiation. At $24 \mathrm{~h}$ after the last UVA irradiation, total RNAs were extracted using a RNeasy Mini kit (Qiagen, CA, USA) according to the manufacturer's protocol. Total RNAs were then used for singlestranded cDNA synthesis using ReverTra Ace qPCR RT Master Mix. Quantitative real-time PCR (qPCR) was performed using the Kapa SYBR FAST with an Eco Real-Time PCR System (Illumina, CA, USA). The following primer sets for COL1A1, MMP-1 and glyceraldehyde 3-phosphate dehydrogenase (GAPDH), as a housekeeping gene, were used: COL1A1 forward, 5'-CCCGGGTTTCAGAGACAACTTC-3', and reverse, 5'-TCCACA TGCTTTATTCCAGCAATC-3', MMP-1 forward, 5'- AAGGTGGACCAAC A A T T T C A G A - 3', and reverse, 5' TGAAGGTGTAGCTAGGGTACATCAA-3', GAPDH forward, 5'-GCACCGTAAGGCTGAGAAC-3', and reverse, 5'-TGGTGAAGACGCCAGTGGA-3’.

\subsection{Western blotting}

Western blotting (WB) of MMP-1 was performed to determine levels of protein expression. NHDFs were exposed to UVA at a dose of $10 \mathrm{~J} / \mathrm{cm}^{2}$ once a day for 3 successive days. NHDFs were cultured in DMEM containing 5\% FBS in the presence or absence of OC or RA for $24 \mathrm{~h}$ after each UVA irradiation. At $48 \mathrm{~h}$ after the last UVA irradiation, supernatants of cells were collected and were mixed with equivalent volumes of sample buffer solution (FUJIFILM Wako Pure Chemical, Osaka, Japan). Ten $\mu \mathrm{L}$ of each mixture were subjected to $10 \%$ gel SDS-PAGE and were blotted to PVDF membranes (Bio Rad, CA, USA). After blocking with $1 \%$ BSA, the membranes were incubated with anti-MMP-1 antibody $(1: 1000)$. After washing, the membranes were further incubated with anti-goat IgG antibody conjugated to $\operatorname{HRP}(1: 1000)$. After washing, MMP-1 was visualized with Ez WestBlue and was quantified using Image J.

\subsection{ELISA assay}

Type I collagen protein levels in the supernatants were measured using an ELISA assay as follows: The supernatants were coated on the bottom of each well in ELISA assay plates (H-plate, Sumitomo Bakelite, Tokyo, Japan)at $4{ }^{\circ} \mathrm{C}$ overnight. After blocking with $1 \% \mathrm{BSA}$, the plates were incubated with anti-collagen I antibody conjugated to 
biotin (1:5000) at $4^{\circ} \mathrm{C}$ overnight and were subsequently incubated with streptavidin- $\operatorname{HRP}(1: 500)$ at room temperature for $1 \mathrm{~h}$. After washing, the plates were reacted with $0.5 \mathrm{mg} /$ $\mathrm{mL}$ ABTS in $0.1 \mathrm{mM}$ sodium citrate buffer $(\mathrm{pH} 4.0)$ in the presence of a small amount of $30 \% \mathrm{H}_{2} \mathrm{O}_{2}$ at $37^{\circ} \mathrm{C}$ for 20 min. Type I collagen was quantified by measuring the absorbance at $405 \mathrm{~nm}$ with a Multiskan FC (MA, USA). Protein contents in cells were quantified with a BCA protein assay kit after lysing with PBS containing 0.5\% Triton X-100. Amounts of type I collagen protein are expressed as pg type I collagen/ $\mu$ g protein.

\subsection{Immunofluorescence staining of collagen fibers}

NHDFs were irradiated with UVA at a dose of $10 \mathrm{~J} / \mathrm{cm}^{2}$ once a day for 3 successive days. NHDFs were cultured in DMEM containing $5 \%$ FBS in the presence or absence of OC or RA for $24 \mathrm{~h}$ after each UVA irradiation. After the final UVA irradiation, the medium was changed to fresh DMEM supplemented with 5\% FBS for $2 \mathrm{w}$. Cells were fixed with $4 \%$ paraformaldehyde for 15 min at room temperature. After blocking with 1\% BSA, cells were incubated with anti-type I collagen antibody (1:500) at $4{ }^{\circ} \mathrm{C}$ overnight and were then further incubated with anti-rabbit IgG $(\mathrm{H}+\mathrm{L})$ antibody-conjugated to Alexa Fluor 594 (1:1000). Nuclei were stained with $2 \mu \mathrm{M}$ Hoechst 33342. Images of fibers were obtained using a Floid Cell Imaging Station and fiber formation was determined and is expressed as the percentage of fiber area in the whole image using corneocytometry software (CIEL, Tokyo, Japan).

\subsection{Statistics}

All data are expressed as means \pm standard deviations. Comparisons between two groups were performed using paired t tests. A $p$-value of less than 0.05 is defined to be statistically significant.

\section{Results}

\subsection{Chemical scavenging properties of OC and RA against ROS}

In order to clarify the scavenging properties of OC and RA against ROS, $\mathrm{IC}_{50}$ values for OC and RA to scavenge $\cdot \mathrm{O}_{2}{ }^{-}$, $\cdot \mathrm{OH}, \mathrm{LOO} \cdot$ and ${ }^{1} \mathrm{O}_{2}$ were examined using the ESR spin trapping method. The results are shown in Table 1. Both OC and RA scavenged every type of ROS tested, however they showed lower scavenging properties against all types of ROS except LOO - compared with ascorbic acid, DL- $\alpha$-tocopherol and $\mathrm{NaN}_{3}$ used as positive controls.

\subsection{Biological effects of OC and RA to reduce intracellu- lar ROS and CP accumulation after UVA irradiation}

UVA caused oxidative stress intracellularly by increasing levels of CPs following the elevation of intracellular ROS. Treatment with OC or RA immediately after UVA irradiation significantly reduced levels of intracellular ROS (Fig. 1a) and decreased levels of intracellular CPs (Fig. 1b, c), which are footprints of the effects of ROS. These results indicated that treatment with OC or RA after UVA exposure alleviated intracellular oxidative stress. Furthermore, the effects of OC and RA on the accumulation of intracellular CPs were examined using repetitively UVA irradiated fibroblasts as a model of chronic UVA exposure of the skin. Repetitive UVA irradiation of NHDFs once a day for 3 successive days resulted in the accumulation of CPs intracellularly, but NHDFs treated with OC or RA immediately after each UVA irradiation had lower levels of intracellular CPs (Fig. 2). These results indicated that OC and RA reduced intracellular oxidative stress initiated by UVA irradiation. This suggested that RA is an active substance in OC, because it showed similar effects to OC despite its lower concentration than in OC.

\subsection{Effects of OC and RA on the synthesis and degrada- tion of collagen}

The restoration effects of $\mathrm{OC}$ and RA on the imbalance of the synthesis and degradation of collagen caused by repetitive UVA irradiation were examined focusing on the behaviors of type I collagen and MMP-1. Repetitive UVA irradiation changed the status of MMP-1 and type I collagen by increasing levels of MMP-1 (Fig. 3) and decreasing levels of type I collagen (Fig. 4) at both the mRNA and protein levels. Although repetitive UVA irradiation increased the protein expression level of pro-MMP-1, the active form of MMP-1 was not detected. OC and RA caused a significant suppression of MMP-1 stimulated by repetitive UVA irradiation (Fig. 3). On the other hand, both OC and RA gave conflicting results on mRNA expression and protein synthesis

Table 1

\begin{tabular}{|c|c|c|c|c|c|c|c|c|c|c|c|c|}
\hline & $\mathrm{IC}_{50}(\mu \mathrm{g} / \mathrm{mL})^{\cdot \mathrm{O}_{2}^{-}}$ & & & $\mathrm{IC}_{50}(\mu \mathrm{g} / \mathrm{mL})$ & & & $\begin{array}{r}\mathrm{LOO} \\
\mathrm{IC}_{50}(\mu \mathrm{g} / \mathrm{mL})\end{array}$ & & & $\mathrm{IC}_{50}(\mu \mathrm{g} / \mathrm{mL}){ }^{{ }^{1} \mathrm{O}_{2}}$ & & \\
\hline & Mean \pm S.D. & $\mathrm{p}^{1}$ & $\mathrm{p}^{2}$ & Mean \pm S.D. & $\mathrm{p}^{1}$ & $\mathrm{p}^{2}$ & Mean \pm S.D. & $\mathrm{p}^{1}$ & $\mathrm{p}^{2}$ & Mean \pm S.D. & $\mathrm{p}^{1}$ & $\mathrm{p}^{2}$ \\
\hline $\mathrm{OC}$ & $873.6 \pm 92.7$ & $* * *$ & $*$ & $285.5 \pm 8.1$ & $* * *$ & $* * *$ & $213.0 \pm 16.0$ & $* * *$ & $* * *$ & $273.1 \pm 15.0$ & $* *$ & N.D. \\
\hline RA & $650.5 \pm 83.7$ & $* * *$ & & $245.4 \pm 4.6$ & $* * *$ & & $110.5 \pm 7.5$ & $* * *$ & & $181.3 \pm 14.9$ & $* *$ & \\
\hline$L$-ascorbic acid & $79.6 \pm 49.0$ & & & $69.8 \pm 0.8$ & & & & & & & & \\
\hline DL- $\alpha$ tocopherol & & & & & & & $803.6 \pm 40.5$ & & & & & \\
\hline $\mathrm{NaN}_{3}$ & & & & & & & & & & $179.4 \pm 11.7$ & & \\
\hline
\end{tabular}


(a)

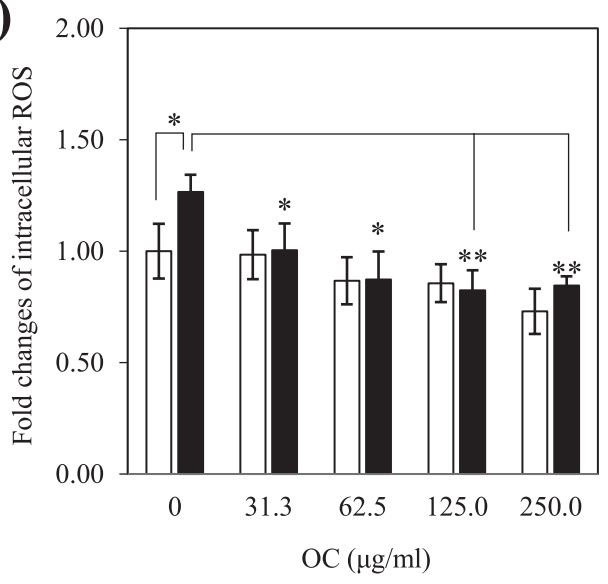

(b)

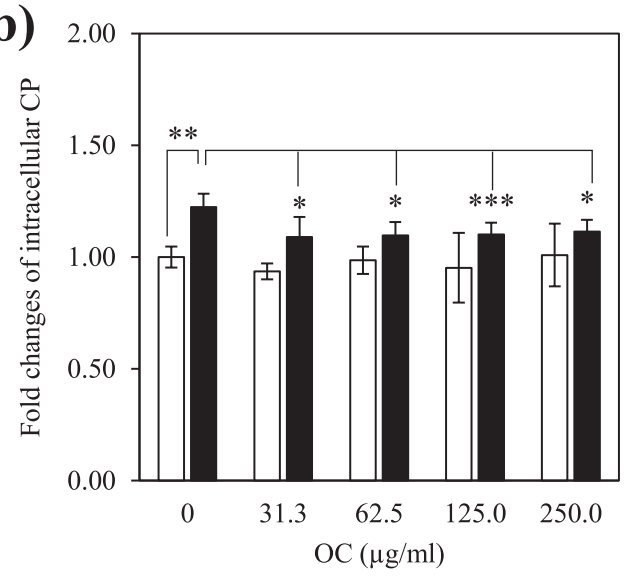

(c)
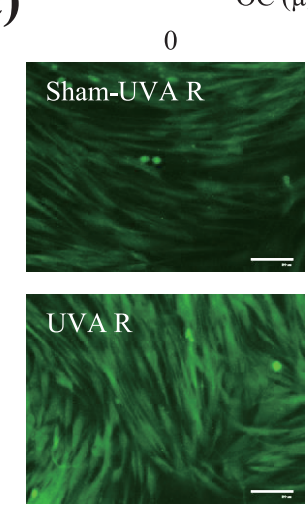
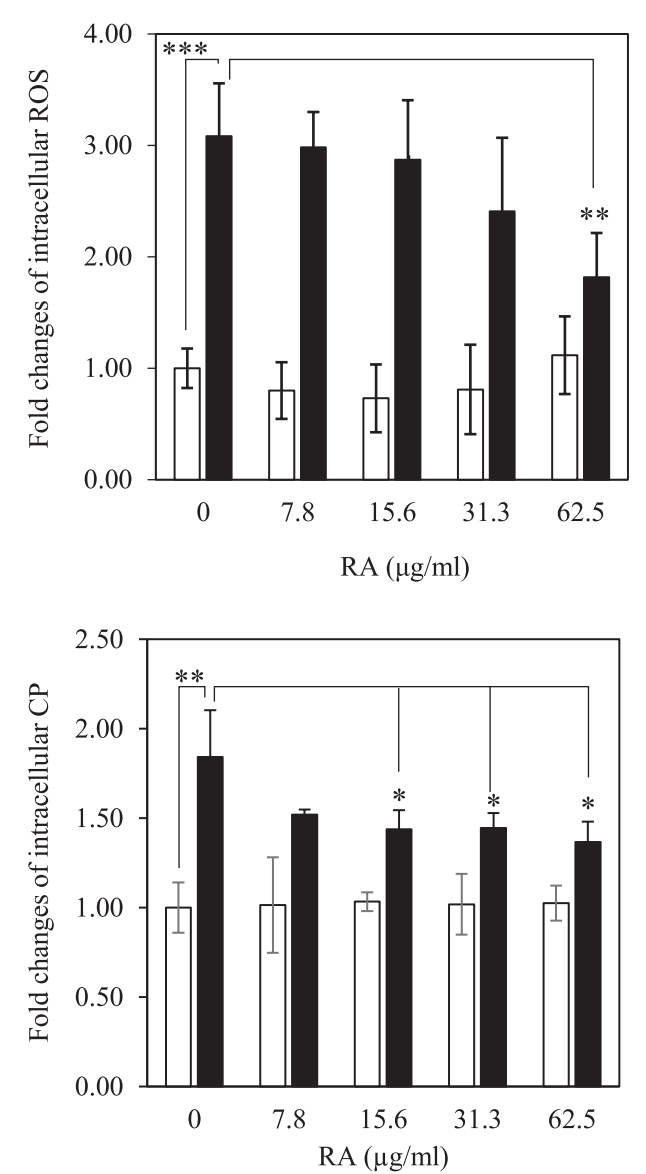

$\mathrm{RA}(\mu \mathrm{g} / \mathrm{mL})$

0
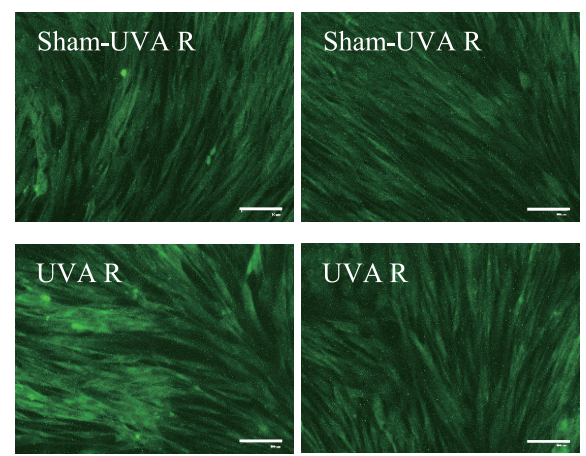

Fig. 1 Suppressive effects of OC and RA on intracellular levels of ROS and CPs elevated by a single UVA irradiation.

(a) After loading with $\mathrm{H}_{2}$ DCFDA, NHDFs were irradiated with UVA at a dose of $10 \mathrm{~J} / \mathrm{cm}^{2}$ and were then cultured in the presence or absence of OC or RA for $6 \mathrm{~h}$. Intracellular ROS levels were determined by measuring fluorescence

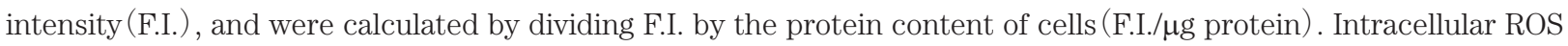
levels are expressed as fold changes normalized with intracellular ROS levels of untreated sham-UVA irradiated NHDFs. Data are expressed as means \pm standard deviations (OC: $n=3, R A: n=4)$. UVA irradiation is abbreviated as UVA R. $\square$; sham-UVA R, $\square$; UVA R. (b) After UVA irradiation, cells were cultured for $24 \mathrm{~h}$ in the presence or absence of OC or RA. Intracellular CPs levels were quantified by measuring F.I. after fluorescence labeling with FTSC, and were calculated as value of the F.I. divided by the F.I. of nuclei stained with Hoechst 33342. Intracellular CPs levels are expressed as fold changes normalized with intracellular CPs of untreated sham-UVA irradiated NHDFs. Data are expressed as means \pm standard deviations $(\mathrm{n}=4)$. $\square$; sham-UVA R, $\square$; UVA R. (c) Representative images of intracellular CPs. Scale bars: $100 \mu \mathrm{m}$. Significance; $* p<0.05, * * p<0.01$ and $* * * p<0.001$. 
(a)

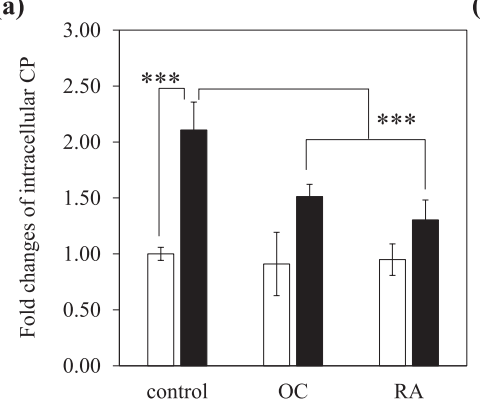

(b)

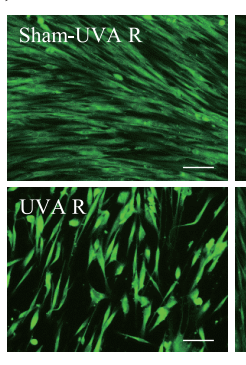

$\mathrm{OC}$

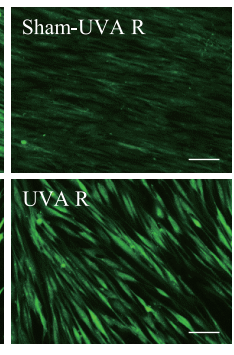

RA

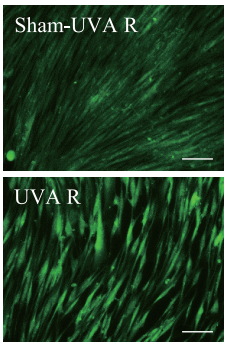

Fig. 2 Suppressive effects of OC and RA against intracellular CPs in NHDFs exposed to repetitive UVA irradiation.

NHDFs were irradiated with UVA at a dose of $10 \mathrm{~J} / \mathrm{cm}^{2}$ once a day for 3 successive days. Cells were cultured in the presence or absence of OC $(250 \mu \mathrm{g} / \mathrm{mL})$ or RA $(62.5 \mu \mathrm{g} / \mathrm{mL})$ after each UVA irradiation, and were further cultured for $24 \mathrm{~h}$ after the final irradiation. After fixation with cold methanol, intracellular CPs and nuclei were detected by fluorescence labeling with FTSC and Hoechst 33342, respectively. (a) Intracellular CPs were quantified by measuring F.I. after fluorescence labeling with FTSC, calculated as the value of F.I. divided by the F.I. of nuclei stained with Hoechst 33342. Intracellular CPs levels are expressed as fold changes normalized with intracellular CPs of untreated sham-UVA irradiated NHDFs. UVA irradiation is abbreviated as UVA R. Data are expressed as means \pm standard deviations $(n=5)$. $\square$; sham-UVA R, $\square$; UVA R. (b) Representative images of intracellular CPs. UVA irradiation is abbreviated as UVA R. Scale bars: $100 \mu \mathrm{m}$. Significance; *** $p<0.001$.

(a)

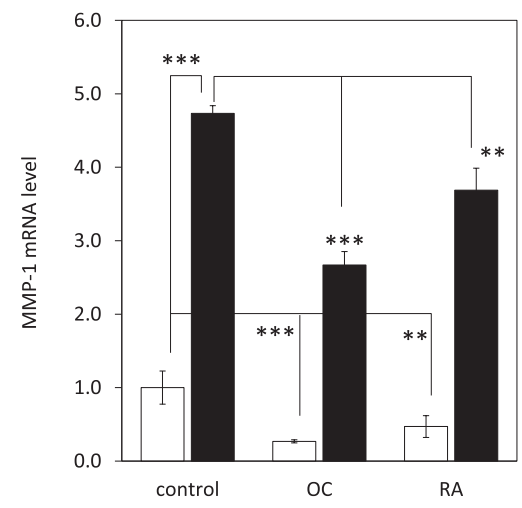

(b)

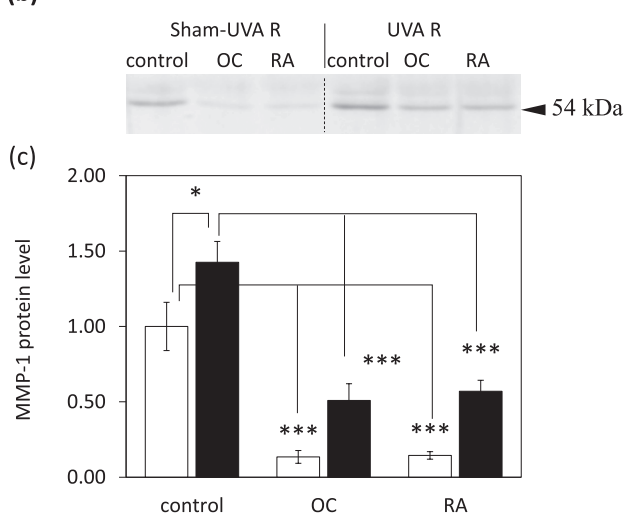

Fig. 3 Restorative effects of OC and RA on MMP-1 altered by repetitive UVA irradiation.

NHDFs were irradiated with UVA at a dose of $10 \mathrm{~J} / \mathrm{cm}^{2}$ once a day for 3 successive days. Cells were cultured in the presence or absence of OC $(250 \mu \mathrm{g} / \mathrm{mL})$ or RA $(62.5 \mu \mathrm{g} / \mathrm{mL})$ after each UVA irradiation, and were further cultured for $24 \mathrm{~h}$ (mRNA) or $48 \mathrm{~h}$ (protein) after the final irradiation. (a) mRNA expression levels of MMP-1 induced by UVA irradiation in NHDFs. mRNA expression levels are expressed as means \pm standard deviations $(n=3)$. UVA irradiation is abbreviated as UVA R. $\square$; sham-UVA R, $\square$; UVA R. (b) Representative images of WBs of MMP-1 proteins secreted from NHDFs irradiated with UVA. (c) MMP-1 levels were digitalized from images of WBs with Image J, and are expressed as means \pm standard deviations $(\mathrm{n}=3) . \square$; sham-UVA R, $\square$; UVA R. Significance; $* p<0.05, * * p<0.01$ and $* * * p<0.001$.

levels of type I collagen in sham-UVA irradiated NHDFs (Fig. 4). Although treatment with OC or RA increased the mRNA expression level of COL1A1 (Fig. 4a), the level of type I collagen protein in NHDFs treated with OC or RA did not show any increase (Fig. 4b). However, treatment with OC or RA significantly restored protein levels of type I collagen which were decreased by repetitive UVA irradiation (Fig. 4b).

\subsection{The effects of OC and RA on the formation of colla-} gen fibers by NHDFs exposed repetitively to UVA

NHDFs produced type I collagen fibers during $14 \mathrm{~d}$ of culture, but repetitive UVA irradiation decreased the formation of type I collagen fibers. The restoration effects of OC and RA on collagen fiber formation by NHDFs following repetitive UVA irradiation were then examined. Treatment with OC or RA significantly improved the decreased formation of type I collagen fibers induced by repetitive UVA irradiation (Fig. 5). 
(a)

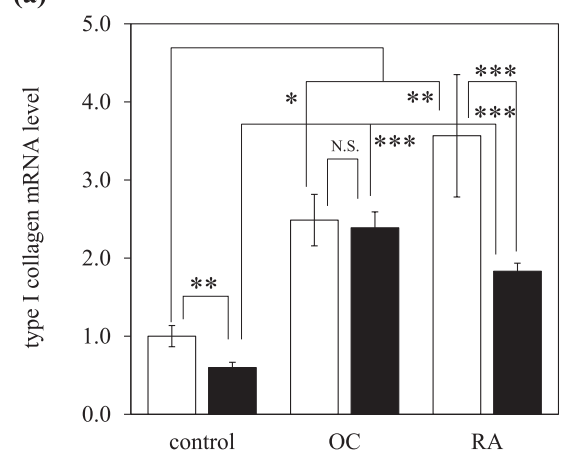

(b)

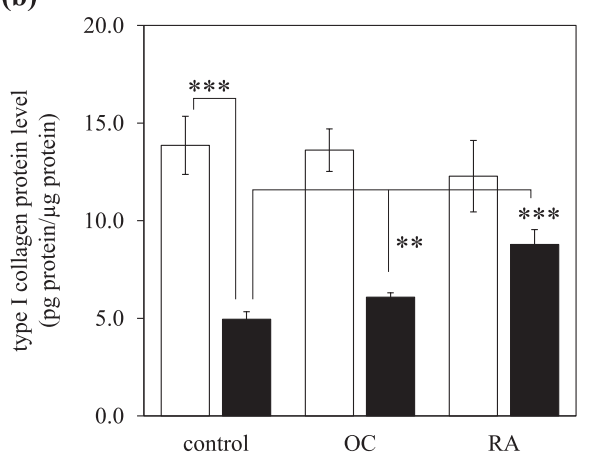

Fig. 4 Restorative effects of OC and RA on type I collagen altered by repetitive UVA irradiation.

NHDFs were irradiated with UVA at a dose of $10 \mathrm{~J} / \mathrm{cm}^{2}$ once a day for 3 successive days. Cells were cultured in the presence or absence of OC $(250 \mu \mathrm{g} / \mathrm{mL})$ or RA $(62.5 \mu \mathrm{g} / \mathrm{mL})$ after each UVA irradiation, and were further cultured for $24 \mathrm{~h}$ (mRNA) or $48 \mathrm{~h}$ (protein) after the final irradiation. (a)mRNA expression levels of type I collagen induced by UVA irradiation are expressed as means \pm standard deviations $(n=4)$. UVA irradiation is abbreviated as UVA R. $\square$; sham-UVA R, $\square$; UVA R. (b) Protein levels of type I collagen were quantified with ELISA, and are expressed as means \pm standard deviations $(\mathrm{n}=4) . \square$; sham-UVA R, $\square$; UVA R. Significance; $* p<0.05, * * p<0.01$ and $* * * p<$ 0.001 .

(a)

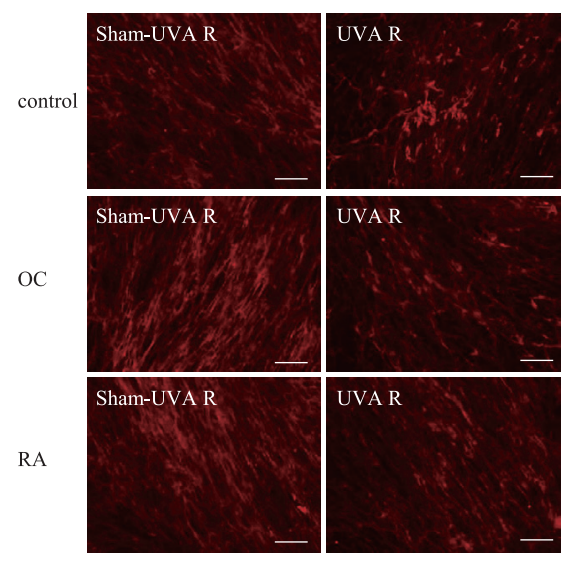

(b)

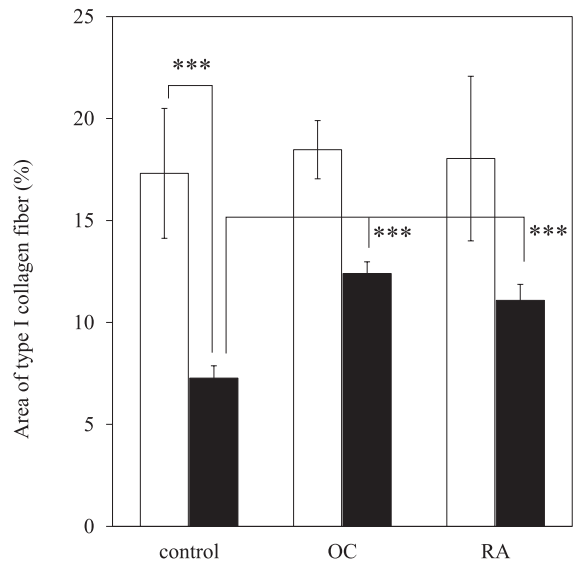

Fig. 5 Effects of OC and RA on type I collagen fiber formation by repetitive UVA irradiation.

NHDFs were irradiated with UVA at a dose of $10 \mathrm{~J} / \mathrm{cm}^{2}$ once a day for 3 successive days. Cells were cultured in the presence or absence of OC $(250 \mu \mathrm{g} / \mathrm{mL})$ or RA $(62.5 \mu \mathrm{g} / \mathrm{mL})$ after each UVA irradiation and then were further cultured for $2 \mathrm{w}$. After fixation with $4 \%$ formaldehyde, collagen fibers were immunostained with anti-type I collagen antibody and anti-rabbit IgG $(\mathrm{H}+\mathrm{L}$ ) antibody-conjugated Alexa Fluor 594. (a) Representative images of collagen fibers, (b) Areas of type I collagen fibers were digitalized with corneocytometry $(n=3)$. Scale bar: $100 \mu$ m. Areas of type I collagen fibers are expressed as means \pm standard deviations. UVA irradiation is abbreviated as UVA R. $\square$; shamUVA R, $\square$; UVA R. Significance; *** $p<0.001$.

\section{Discussion}

Exposure to sunlight, which includes UVB and UVA, initiates and promotes photoaged skin characterized by deep wrinkles and sags. It has been demonstrated that a fragile dermal structure, including the loss of collagen fibers due to the altered function of fibroblasts, is responsible for the formation of wrinkles and sags. The loss of collagen fibers in the upper dermis is caused by an imbalance between the synthesis and degradation of collagen fibers triggered by ROS. On the other hand, among the different types of UV, it is recognized that UVA is the major generator of ROS in fibroblasts and in the dermis, because UVB hardly penetrates into the dermis ${ }^{1)}$. The mechanism by which ROS causes the imbalance between the synthesis and degradation of collagen fibers has been well demonstrated as follows; ROS increases the production of Cyr61/CCN-1 (cysteine-rich angiogenic inducer 61), which is an internal suppressor of collagen synthesis ${ }^{13)}$, and MMP-1, which is a collagenase that degrades type I fibrillar collagens ${ }^{14)}$, through the activation of epidermal growth factor-receptor 
signaling ${ }^{15)}$. Furthermore, ${ }^{1} \mathrm{O}_{2}$ generated through the photosensitization reaction by UVA stimulates the biosynthesis of MMP-1 in fibroblasts through the signaling of IL-1 and IL- $6{ }^{16)}$. Summarizing those facts, the elevation of intracellular ROS, for example, by stimulation with UVA, results in the depletion of collagen fibers due to an imbalance of its synthesis and degradation in the dermis. Thus, in order to estimate the potential of an effective anti-photoaging material, restoration of the imbalance between collagen synthesis and MMP-1 through the reduction of intracellular ROS is usually examined. Thus, in this study, the potential of OC, which contains RA, as an anti-photoaging material was investigated focusing on the restoration of the imbalance between the synthesis and degradation of collagen based on the reduction of intracellular ROS and the formation of collagen fibers impaired by repetitive UVA irradiation. Regarding UVB, it is presumed that substances secreted from UVB-exposed keratinocytes are causative factors in the imbalance between the synthesis and degradation of collagen fibers, since fibroblasts are not directly exposed to UVB. The effects of OC or RA on the altered function of fibroblasts initiated by substances secreted from UVB-exposed keratinocytes will be reported elsewhere after continuing the study.

Although it has been reported that OC contains antioxidant components in addition to RA, such as linalool, eugenol, methyl chavicol, methyl cinnamate, ferulate methyl eugenol, triterpenoids and steroidal glycoside ${ }^{17)}$, OC and RA did not show higher scavenging abilities against ROS from the chemical aspect (Table 1). However, from the biological aspect, both OC and RA showed significant reductions of levels of ROS and intracellular CPs in fibroblasts that were elevated by a single exposure to UVA (Fig. 1). Furthermore, OC and RA showed significant reductions of CPs in repetitively UV irradiated fibroblasts, which is a model of chronic sun-exposure (Fig. 2). The sum of these results indicates that $\mathrm{OC}$ and $\mathrm{RA}$ reduce intracellular oxidative stress biologically regardless of their relatively poor scavenging abilities from the chemical aspect.

Regarding the imbalance between collagen synthesis and MMP-1 production in fibroblasts repetitively irradiated with UVA, OC and RA significantly restored both of them at the protein level(Fig. 3, 4). However, OC and RA gave conflicting results between mRNA and protein levels involved in the synthesis of type I collagen. Despite upregulating the mRNA expression level of COL1A1 in sham-UVA irradiated fibroblasts, OC and RA did not show any changes in the level of type I collagen protein (Fig. 4). Since type I collagen is composed of COL1A1 and COL1A2 chains ${ }^{18)}$, it might be presumed that OC and RA did not have an effect on the mRNA expression level of COL1A2. However, both $\mathrm{OC}$ and RA restored the imbalance between the synthesis and degradation of type I collagen induced by repetitive UV irradiation, because protein levels are a more critical issue than mRNA levels. Furthermore, OC and RA significantly improved collagen fiber formation that was impaired by repetitive UVA irradiation(Fig. 5). Collagen fibers are constructed by the cross-linking of triple-helical collagen peptides, COL1A1 and COL1A2, by enzymatic reaction with lysyl oxidase (LOX) after secretion ${ }^{19)}$. In a preliminary examination, we found that fibroblasts exposed to oxidative stress had a downregulation of LOX mRNA expression (data not shown). The restoration effects of OC and RA on collagen fiber formation might be involved in improving the change of LOX protein. We will continue further studies to demonstrate the restoration effects of OC and RA on collagen fiber formation in more detail and will report those elsewhere.

\section{Conclusion}

This study confirmed that treatment with OC or RA reduced levels of intracellular ROS and intracellular CPs in UVA irradiated fibroblasts, which resulted in the improvement of collagen fibers formed by UVA irradiated fibroblasts. It was considered that the reduction of intracellular oxidative stress by OC and RA is responsible for those improvements. In addition, based on the effective concentrations of OC and RA, the results suggest that RA is one of the active components in OC.

Taken together, these results demonstrate that OC, which contains RA, improves or prevents photoaged skin by restoring collagen fiber formation that is impaired by repetitive UVA irradiation.

\section{References}

1) Battie, C.; Verschoore, M. Cutaneous solar ultraviolet exposure and clinical aspects of photodamage. Indian J. Dermatol. Venereol. 78 (Suppl 1), S9-S14(2012).

2) Lan, C.E.; Hung, Y.T.; Fang, A.H.; Ching-Shuang, W. Effects of irradiance on UVA-induced skin aging. $J$. Dermatol. Sci. 94, 220-228(2019).

3) Larroque-Cardoso, P.; Camaré, C.; Nadal-Wollbold, F.; Grazide, M.H.; Pucelle, M.; Garoby-Salom, S.; Bogdanowicz, P.; Josse, G.; Schmitt, A.M.; Uchida, K.; Zarkovic, K.; Salvayre, R.; Nègre-Salvayre, A. Elastin modification by 4-hydroxynonenal in hairless mice exposed to UV-A. Role in photoaging and actinic elastosis. $J$. Invest. Dermatol. 135, 1873-1881 (2015).

4) Braverman, I.M.; Fonferko, E. Studies in cutaneous aging: I. The elastic fiber network. J. Invest. Dermatol. 78, 434-443 (1982).

5) Rittié, L.; Fisher, G.J. Natural and sun-induced aging of human skin. Cold Spring Harbor Perspect. Med. 5, a015370 (2015). 
6) Quan, T.; Qin, Z.; Xia, W.; Shao, Y.; Voorhees, J.J.; Fisher, G.J. Matrix-degrading metalloproteinases in photoaging. J. Invest. Dermatol. Symp. Proc. 14, 20-24 (2009).

7) Fisher, G.J.; Voorhees, J.J. Molecular mechanisms of photoaging and its prevention by retinoic acid: Ultraviolet irradiation induces MAP kinase signal transduction cascades that induce Ap-1-regulated matrix metalloproteinases that degrade human skin in vivo. J. Invest. Dermatol. Symp. Proc. 3, 61-68(1998).

8) Bäumler, W.; Regensburger, J.; Knak, A.; Felgenträger, A.; Maisch, T. UVA and endogenous photosensitizers the detection of singlet oxygen by its luminescence. Photochem. Photobiol. Sci. 11, 107-117(2012).

9) Valencia, A.; Kochevar, I.E. Nox1-based NADPH oxidase is the major source of UVA-induced reactive oxygen species in human keratinocytes. J. Invest. Dermatol. 128, 214-222 (2008).

10) Yamawaki, Y.; Mizutani, T.; Okano, Y.; Masaki, H. The impact of carbonylated proteins on the skin and potential agents to block their effects. Exp. Dermatol. 28 (Suppl 1), 32-37 (2019).

11) Jayasinghe, C.; Gotoh, N.; Aoki, T.; Wada, S. Phenolics composition and antioxidant activity of sweet basil (Ocimum basilicum L.). J. Agric. Food Chem. 51, 4442-4449 (2003).

12) Fernando, P.M.; Piao, M.J.; Kang, K.A.; Ryu, Y.S.; Hewage, S.R.; Chae, S.W.; Hyun, J.W. Rosmarinic acid attenuates cell damage against UVB radiation-induced oxidative stress via enhancing antioxidant effects in human HaCaT cells. Biomol. Ther. 24, 75-84 (2016).

13) Quan, T.; He, T.; Shao, Y.; Lin, L.; Kang, S.; Voorhees, J.J.; Fisher, G.J. Elevated cysteine-rich 61 mediates aberrant collagen homeostasis in chronologically aged and photoaged human skin. Am. J. Pathol. 169, 482490 (2006).

14) Yucel, T.; Mutnal, A.; Fay, K.; Fligiel, S.E.; Wang, T.; Johnson, T.; Baker, S.R.; Varani, J. Matrix metalloproteinase expression in basal cell carcinoma: Relationship between enzyme profile and collagen fragmentation pattern. Exp. Mol. Pathol. 79, 151-160 (2005).

15) Kajanne, R.; Miettinen, P.; Mehlem, A.; Leivonen, S.K.; Birrer, M.; Foschi, M.; Kähäri, V.M.; Leppä, S. EGF-R regulates MMP function in fibroblasts through MAPK and AP-1 pathways. J. Cell. Physiol. 212, 489-497 (2007).

16) Wlaschek, M.; Heinen, G.; Poswig, A.; Schwarz, A.; Krieg, T.; Scharffetter-Kochanek, K. UVA-induced autocrine stimulation of fibroblast-derived collagenase/ MMP-1 by interrelated loops of interleukin- 1 and interleukin-6. Photochem. Photobiol. 59, 550-556 (1994).

17) Zheljazkov, V.D.; Callahan, A.; Cantrell, C.L. Yield and oil composition of 38 basil (Ocimum basilicum L.) accessions grown in Mississippi. J. Agric. Food Chem. 56, 241-245 (2008).

18) Piez, K.A.; Eigner, E.A.; Lewis, M.S. The chromatographic separation and amino acid composition of the subunits of several collagens*. Biochemistry 2, 58-66 (1963).

19) Herchenhan, A.; Uhlenbrock, F.; Eliasson, P.; Weis, M.; Eyre, D.; Kadler, K.E.; Magnusson, S.P.; Kjaer, M. Lysyl oxidase activity is required for ordered collagen fibrillogenesis by tendon cells. J. Biol. Chem. 290, 16440$16450(2015)$. 